Departamento de Ciência e Tecnologia, Secretaria de Ciência, Tecnologia e Insumos Estratégicos, Ministério da Saúde

Correspondência | Correspondence:

Decit - Departamento de Ciência e Tecnologia do Ministério da Saúde

Esplanada dos Ministérios Bloco G sala 845

70058-900 Brasília, DF, Brasil

Texto de difusão técnico-científica do Ministério de Saúde

\section{Encontro Anual da Sociedade Health Technology Assessment International, Brasil, 2011}

\author{
Annual meeting of the Health \\ Technology Assessment International \\ Society, Brazil, 2011
}

\section{INTRODUÇÃO}

A avaliação de tecnologias em saúde (ATS) é um processo abrangente que reconhece os impactos clínicos, sociais e econômicos das tecnologias em saúde, levando em consideração aspectos como eficácia, efetividade, custos, segurança e custo-efetividade. O principal objetivo da ATS é auxiliar na tomada de decisão de gestores, dirigentes e profissionais de saúde quanto à incorporação de tecnologias sanitárias. No Brasil, essas tecnologias incluem medicamentos, equipamentos e procedimentos técnicos, sistemas organizacionais, educacionais, de informação e de suporte. Também incluem programas e protocolos assistenciais por meio dos quais a atenção e os cuidados com a saúde são prestados à população.

O Health Technology Assessment International (HTAi) é uma sociedade científica e profissional internacional voltada para todos aqueles que produzem, utilizam ou se deparam com questões relativas à gestão e à avaliação de tecnologias em saúde. Ela atua como um fórum neutro para colaboração e troca de informações e conhecimento em ATS, abrangendo todos os atores interessados - pesquisadores, dirigentes e tomadores de decisão, prestadores de serviços de saúde, pacientes/ usuários e profissionais da indústria e da academia. Com membros de 59 países localizados nos seis continentes, o HTAi é uma sólida rede global na área de ATS.

O encontro anual do HTAi é a principal conferência mundial que discute bases conceituais, métodos e aplicações da ATS como forma de ampliar a qualidade e acesso a tecnologias efetivas, seguras e eficientes. Os encontros são organizados por uma instituição anfitriã, apoiada por um Comitê Internacional de Programa Científico (International Scientific Program Committee - ISPC) e por um Comitê de Organização
Local (Local Organizing Committee - LOC). A seleção das organizações anfitriãs ocorre por meio de um processo internacional que avalia o mérito da instituição proponente. A Secretaria de Ciência, Tecnologia e Insumos Estratégicos do Ministério da Saúde, por meio do Departamento de Ciência e Tecnologia (Decit), enviou em 2008 proposta para sediar o evento no ano de 2011, aprovada em junho de 2009. A proposta contou com o apoio de sociedades e associações científicas nacionais e internacionais, incluindo a Associação Brasileira de Pós-Graduação em Saúde Coletiva, o Council on Health Research for Development e a Fundação de Apoio a Pesquisa do Estado do Rio de Janeiro.

As atribuições do Decit como organizador do HTAi 2011 incluíram as seguintes atividades: i) composição do ISPC, encarregado de orientar a composição do programa científico, e do Comitê Revisor, responsável por elaborar regras e analisar os trabalhos científicos submetidos para apresentação durante o evento; ii) organização das reunião do LOC e orientação da divulgação no âmbito do Comitê Consultivo (Advisory Committee); e iii) coordenação do comitê executivo responsável por organizar e implementar todas as ações relativas à organização do evento.

Essa foi a primeira vez que o encontro anual do HTAi ocorreu fora do hemisfério norte. O Decit/MS, para realização do evento, contou com a parceria da Fundação Oswaldo Cruz, da Agência Nacional de Saúde Suplementar, da Agência Nacional de Vigilância Sanitária, do Centro Cochrane do Brasil/Universidade Federal de São Paulo, da Organização Pan Americana de Saúde, de instituições integrantes da Rede Brasileira de Avaliação de Tecnologias em Saúde (Rebrats), ${ }^{a}$ de hospitais filantrópicos de excelênciab e com países membros e associados do Mercosul.

\footnotetext{
a Universidade Estadual do Rio de Janeiro, Universidade Federal do Rio de Janeiro, Universidade Federal do Rio Grande do Sul, Universidade de São Paulo, Universidade Federal de São Paulo, Instituto Fernandes Figueira, Instituto Nacional de Cardiologia, Instituto Nacional de Traumatologia e Ortopedia e Escola Nacional de Saúde Pública.

b Instituto de Educação e Ciências do Hospital Alemão Oswaldo Cruz, Instituto de Ensino e Pesquisa do Hospital do Coração. c Argentina, Uruguai, Paraguai, Chile.
} 


\section{TEMÁTICA DO EVENTO: ATS PARA SUSTENTABILIDADE DOS SISTEMAS DE SAÚDE}

Após os debates na Conferência das Nações Unidas para o Meio Ambiente e o Desenvolvimento, no Rio de Janeiro, em 1992 (ECO-92), e em encontros mundiais subseqüentes, a sustentabilidade dos sistemas de saúde passou a ser considerada a preocupação principal da humanidade no século 21. A maior parte dos debates girou em torno do clima e do meio ambiente, mas a sustentabilidade dos sistemas humanos passou também a representar um desafio importante.

As demandas sobre os sistemas de saúde aumentam à medida que as populações crescem e/ou envelhecem e que os avanços científicos e as expectativas da população com relação à saúde e à qualidade de vida aumentam. Entretanto, ainda que novas tecnologias permitam que doenças antes incuráveis sejam tratadas, mesmo que muitas vezes a custos elevados, várias das principais causas de doenças globais permanecem sem tratamento efetivo.

Qualidade, eqüidade, acesso e cobertura universais são preocupações crescentes para todos os países - e os gastos em saúde sofrem constantes pressões e controles. $\mathrm{O}$ uso apropriado de tecnologias e a alocação de recursos a fim de maximizar o valor obtido é componente-chave da sustentabilidade dos sistemas de saúde. Ao fornecer informações necessárias para que a tomada de decisões seja baseada em evidências científicas de forma válida, transparente e responsável, a ATS desempenha papel essencial para apoiar os processos de melhoria da qualidade e de cobertura em direção à sustentabilidade. Nesse sentido, o Encontro Anual HTAi 2011 teve como tema central a sustentabilidade dos sistemas de saúde e o papel da ATS nesse contexto.

\section{DESTAQUES DA PROGRAMAÇÃO CIENTÍFICA}

O $8^{\circ}$ Encontro Anual da Sociedade HTAi, realizado de 25 a 29 de junho de 2011, no Rio de Janeiro, contou com a participação de 1.075 congressistas de 51 países. O Ministro da Saúde, Alexandre Padilha, abriu o evento com o panorama da saúde no Brasil, destacando o importante papel da ATS na priorização de políticas públicas e no processo de tomada de decisões, uma vez que as demandas são infinitas e os recursos disponíveis são limitados. O foco da produção de tecnologias voltadas às ações de atenção primária em saúde pontuou o discurso do ministro como uma das dimensões nas quais o Brasil deverá avançar na discussão sobre tecnologias em saúde. O Ministro Padilha apontou a produção nacional como "decisiva para a sustentabilidade do Sistema Único de Saúde".

A pré-conferência, nos dias 25 e 26 de junho, acolheu minicursos em temas relativos à ATS. As atividades da conferência foram desenvolvidas nos dias 27,28 e 29 de junho. Os trabalhos foram abertos pelo Secretário de Ciência, Tecnologia e Insumos Estratégicos, Carlos Gadelha, com a palestra magna "Saúde, Desenvolvimento e Inovação - Desafios para os Sistemas de Saúde”. Em sua exposição, foram abordadas as rápidas mudanças socioeconômicas que têm transformado a pirâmide social e etária no País - e como elas exigem que haja geração de conhecimento para atender os novos objetivos sociais. O crescimento da classe média, a redução da mortalidade infantil e o envelhecimento da população vão demandar investimentos na produção nacional de pesquisa, desenvolvimento e inovação em saúde, orientados para as macro-regiões. Hoje, a produção nacional não acompanha a demanda por novas tecnologias. $\mathrm{O}$ desafio do Brasil é elevar o financiamento público em saúde para mudar esse cenário. Segundo Gadelha, uma das soluções que o Ministério da Saúde tem encontrado para um maior investimento na produção nacional em saúde no Brasil são as parcerias para o desenvolvimento produtivo: "Elas dão a chance de integrar de forma cooperativa o setor privado e o público. Dessa forma, o governo pauta a agenda produtora nacional, chamando o setor privado a investir onde o Estado não tem capacidade", afirmou.

Ao todo, foram realizadas três sessões plenárias, que trataram dos seguintes temas: "O Desafio: Sustentabilidade dos Sistemas de Saúde no Século 21"; "Progresso: Estudo de Casos sobre o Desenvolvimento e Impacto da Avaliação de Tecnologias em Saúde nos Sistemas de Saúde"; e "Próximos Passos: Desenvolver Avaliação de Tecnologias em Saúde para Sistemas de Saúde Sustentáveis". O evento contou, também, com sessões de painéis - resultantes de trabalhos de especialistas de renome internacional submetidos a processo de seleção; sessões orais - trabalhos científicos submetidos ao processo de seleção e que obtiveram as notas mais altas; e duas sessões de pôsteres.

No âmbito técnico-científico, nos diversos painéis foram apresentados, com destaque para os debates sobre o papel da ATS na avaliação de intervenções terapêuticas, os processos de regulação de listas de cobertura de medicamentos, o papel da ATS na análise de inovações e a retirada de tecnologias (desinvestimento). Debates sobre a implementação de políticas de ATS em países emergentes e a colaboração transcontinental foram importantes para delinear estratégias de consolidação da ATS no mundo, com forte perspectiva de expansão na América Latina.

\section{PERFIL DOS TRABALHOS CIENTÍFICOS}

Os trabalhos científicos apresentados durante o evento foram submetidos a um processo de seleção, aproximadamente cinco meses antes da data da conferência, por um comitê composto por 78 especialistas de 21 
países. Cada resumo científico foi analisado por três especialistas diferentes. A identidade dos autores foi mantida em sigilo na avaliação.

Os trabalhos foram classificados em uma escala de 1 a 5 , cujos critérios foram a relevância do tópico, adequação metodológica e aplicabilidade. Aqueles com pontuações mais altas foram selecionados para apresentação oral. As atribuições das escalas foram combinadas em um único resultado por avaliador e a média dos valores aferidos pelos avaliadores foi usada para determinar a aceitabilidade do trabalho e o modo de apresentação. Não houve limites ao número de trabalhos que um autor ou grupo de autores poderia submeter. No entanto, cada autor poderia apresentar apenas um trabalho em sessões orais ou no máximo dois pôsteres, assegurando, dessa forma, a maximização do número de participantes aptos a apresentar seus trabalhos.

Foram selecionados 828 resumos, sendo os primeiros 170 para apresentações orais $(19,7 \%$ do total submetido) e os demais para apresentação sob a forma de pôster (Figura 1).

A distribuição temática dos resumos selecionados foi a seguinte: $27 \%$ na área de estudos clínicos, $20 \% \mathrm{em}$ estudos econômicos, $26 \%$ em política e prática de saúde, $16 \%$ em métodos e $10 \%$ foram classificados como "outros" (Figura 2).

Os resumos representaram 44 países, tendo garantido a diversidade de experiências. A América Latina e o Caribe apresentaram 499 resumos; seguidos da Europa, com 193; Ásia, com 69; América do Norte, com 48; Oceania, com 13 e África, com seis (Figura 3).

Considerando a América Latina e o Caribe, o país com a maioria de resumos foi o Brasil (438, 91,8\% da região), coerente com o número de resumos científicos submetidos (461), seguido da Argentina, com 16 (3,35\%), e Uruguai, com 13 (2,73\%).

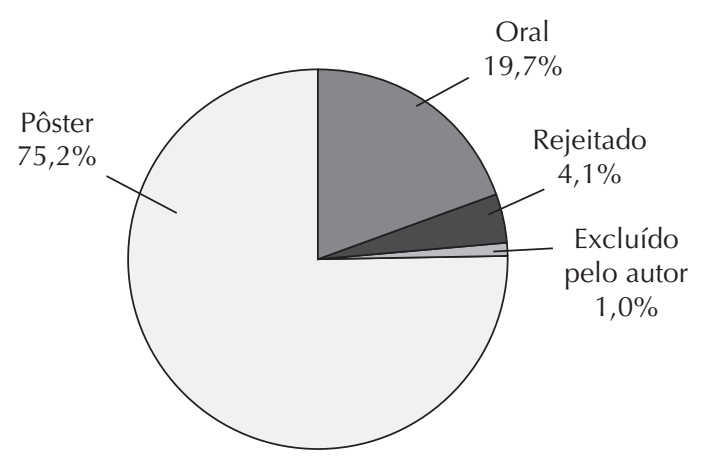

Fonte: Comitê Internacional de Programa Científico e Comitê de Organização Local, 2011

Figura 1. Resumos científicos aprovados.

\section{PREMIAÇÃO}

Foram distribuídos quatro prêmios durante o evento. Dois na categoria pôster - "Cost-Effectiveness Analysis and Budget Impact Assessment: a Graphical Way to Combine the Two for the Aid of Decision Makers", de Mike Paulden, do Canadá, e "Results of Two Systematic Reviews of Methods for Meta-Analysis and Indirect Comparison Used In Existing Systematic Reviews or Potentially Available to Use In Systematic Reviews", de Jacek Walczak, da Polônia.

$\mathrm{Na}$ categoria trabalho científico, os dois vencedores foram "Real Word Effectiveness and Compliance of Hepatitis C Treatment in Brazil: Meta Analysis and Meta Regression", de Carine Raquel Blatt, do Brasil; e "Estimating the SF-6D Value Set for a Southern Brazilian Population", de Luciane Nascimento Cruz, do Brasil.

\section{PARTICIPAÇÃO BRASILEIRA NOS ENCONTROS ANUAIS}

Nos anos anteriores o encontro anual do HTAi ocorreu nos seguintes países: Polônia, Itália, Austrália e Espanha, Canadá, Cingapura e Irlanda. A participação do Brasil tem sido crescente desde o II Encontro Anual do HTAi, em 2005, na Itália. O Ministério da Saúde, por meio do Decit, tem comparecido aos encontros e incentivado a participação de seus técnicos. Como resultado, além da ampliação da equipe que tem participado dos encontros anuais, os técnicos e pesquisadores brasileiros realizaram troca de experiências com a comunidade científica internacional, resultando no aprimoramento das atividades de ATS realizadas pela Coordenação Geral de Avaliação de Tecnologias em Saúde (CGATS) do Ministério da Saúde.

Em 2006, na Austrália, o Decit apresentou três pôsteres; quatro na Espanha, em 2007, e em 2008, no Canadá, além das duas apresentações orais, a equipe da CGATS/

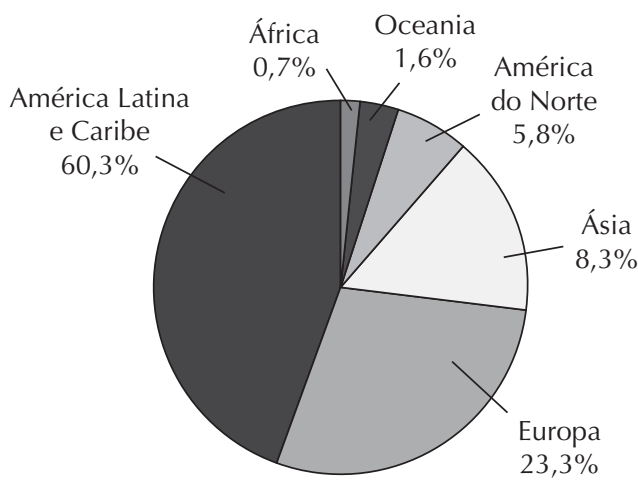

Fonte: Comitê Internacional de Programa Científico e Comitê de Organização Local, 2011

Figura 2. Trabalhos aprovados por região. 


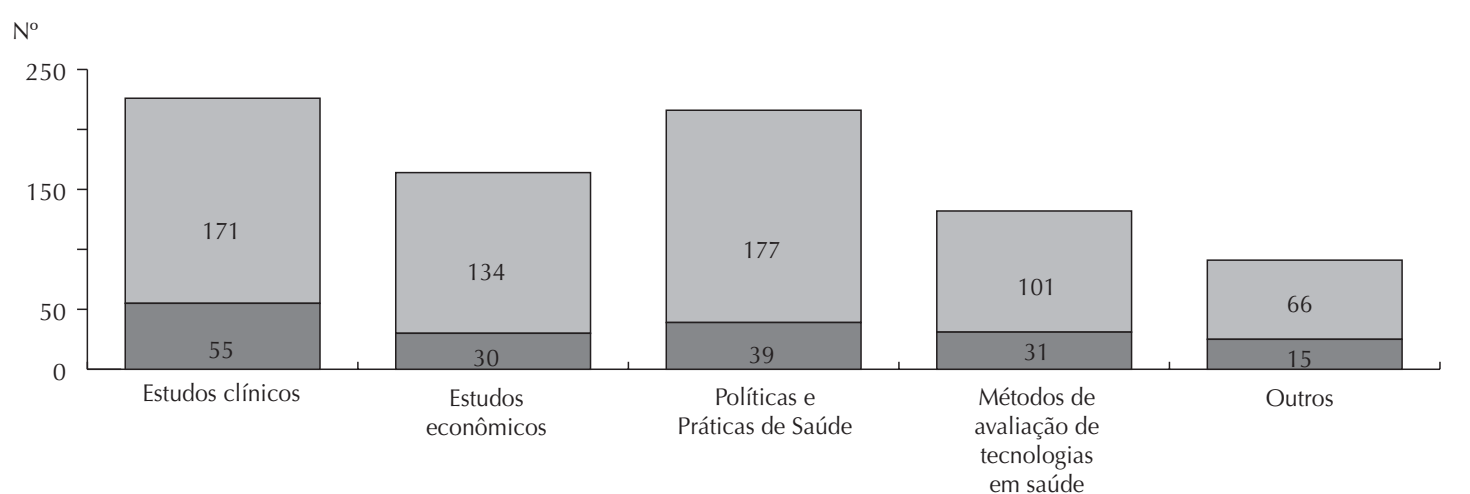

Fonte: Comitê Internacional de Programa Científico e Comitê de Organização Local, 2011

Figura 3. Resumos científicos aprovados por área e tipo de apresentação.

Decit exibiu oito pôsteres e organizou um workshop a convite dos organizadores do evento. Em 2009, em Cingapura, a equipe do Decit participou como autora principal de 13 trabalhos apresentados em pôsteres. $\mathrm{Na}$ Irlanda, em 2010, dez técnicos do Decit apresentaram quinze trabalhos na modalidade pôster. Neste ano, os técnicos do Ministério da Saúde participaram de cinco painéis e foram citados em 34 resumos científicos. Destes, 11 foram apresentados em sessões orais e 24 apresentados em forma de pôster.

\section{REPERCUSSÕES DO ENCONTRO ANUAL HTAi 2011}

O Ministro Alexandre Padilha destacou a importância de se ter uma política de ATS como fator decisivo na qualidade e segurança dos serviços prestados para a população. Assim, com vista a fortalecer o sistema de saúde, o governo brasileiro aprovou lei que estabelece a Comissão Nacional de Incorporação Tecnológica, a qual conta com especialistas e representantes dos governos estaduais e municipais. Destacou ainda o importante papel da Rede Brasileira de Avaliação de Tecnologias em Saúde (Rebrats), que congrega 44 instituições, e a construção de uma agenda de pesquisas capazes de responder aos grandes desafios da saúde no País. Mostrou que o fato de sediar a reunião reforçou a política de ATS do Brasil e propiciou proximidade com seus parceiros internacionais. Informou que o Brasil vai tratar o tema ATS como central na articulação e discussões no âmbito do BRICS (Brasil, Rússia, Índia, China e África do Sul), assim como a importância da produção de inovação tecnológica e o acesso a essa tecnologia.

O Secretário de Ciência, Tecnologia e Insumos Estratégicos, Carlos Gadelha - presidente do HTAi 2011, destacou que a inovação está no eixo central da Política Nacional de Saúde e que o sistema de inovação precisa atender as demandas da saúde. O foco do Governo não está no que se pode pagar, mas em como se pode direcionar a inovação para que se viabilize o acesso à saúde e com a qualidade que a população precisa. A agenda do Governo tem como objetivo viabilizar a inovação a serviço da população brasileira.

O Presidente da Fundação Oswaldo Cruz, Paulo Gadelha, lembrou a importância da ATS para que se possa fazer uma sinergia entre os campos da inovação, da disponibilidade de produtos e insumos e a sustentabilidade de um sistema como o brasileiro. $\mathrm{O}$ apoio ao evento reflete a prioridade política da ATS no País e a maturidade no tema. Lembrou o papel importante da ATS quando se trata de doenças negligenciadas, orientando a aplicação dos recursos.

A Diretora do Departamento de Ciência e Tecnologia do Ministério da Saúde do Brasil, Ana Luiza D’Ávila Viana, destacou que a avaliação e incorporação de tecnologias podem garantir o acesso à saúde aos mais de 190 milhões de brasileiros, a incorporação de inovações tecnológicas pelo parque produtivo nacional, e também ampliar o nosso leque de pesquisas. Lembrou que o Ministério da Saúde é hoje um dos maiores financiadores da pesquisa e da inovação em saúde no Brasil. O cumprimento dos princípios da integralidade e universalidade do sistema de saúde brasileiro depende do tripé acesso por meio de novas tecnologias, produção e expansão do parque produtivo nacional, e ampliação do leque de pesquisas, juntamente com uma política de saúde. Destacou ainda que o Brasil é visto hoje como um país que pode garantir o direito social à saúde com crescimento econômico de grande potencial, e que mostra solidariedade com os outros países da região e da África. Isso confere ao Brasil o protagonismo na agenda internacional, em especial a expansão das relações no eixo Sul-Sul. Segundo ela, a realização da reunião anual HTAi mostrou que o Brasil é líder no estabelecimento de relações que possam garantir o acesso a melhores condições de vida. 
A responsável pela área de ATS do Decit, Flávia Elias, vice-presidente do HTAi 2011, lembrou que a realização de evento de tal envergadura no Brasil é o reconhecimento do esforço empreendido pelo Ministério da Saúde.

Clarisse Petramale, da Comissão Nacional para Incorporação de Tecnologias em Saúde, destacou os importantes investimentos no acolhimento e cuidado com os pacientes.

O Dr. Joseph Matthew, da Índia, destacou a importância do Brasil em sediar tal evento e que os países membros do BRICS observam os trabalhos em desenvolvimento no Brasil para aplicação de suas experiências em seus países.

A Dr. ${ }^{a}$ Laura Sampietro-Colom, Presidente da Sociedade HTAi no período 2009 a 2010-11, destacou que o Brasil cresce não apenas economicamente, mas também socialmente. O País abraçou fortemente a ATS e prova disso é a realização dessa edição da reunião anual HTAi, que pela primeira vez foi realizada na América do Sul. Destacou ainda o papel do Brasil como catalisador e capaz de ajudar outros países a utilizar ATS em seus processos de desenvolvimento, bem como a parceria estabelecida entre o Ministério da Saúde e a sociedade nos últimos três anos para a realização dessa reunião. Mencionou a escolha exitosa dos dois co-presidentes do ISPC, um especialista brasileiro e outro europeu, que foram hábeis na escolha dos temas e propiciaram um equilíbrio nos assuntos abordados; e também a importância que redes, como a Rebrats, desenvolvem na disseminação da ATS. No tocante ao desenvolvimento de recursos humanos, destacou o interesse mostrado por um grande número de jovens profissionais.

O atual Presidente da Sociedade HTAi, Clifford Goodman, chamou a atenção para o sucesso da reunião e destacou o grande número de participantes de vários países da região, metade deles brasileiros. Lembrou que ATS só produz bons resultados quando adaptada às realidades locais, regionais e nacionais. ATS não compreende apenas os aspectos clínicos, mas também econômicos, sociais, éticos, legais e culturais da tecnologia. Nesse sentido, destacou ser importante que o Brasil não apenas adote os métodos de ATS, mas também desenvolva seus próprios parâmetros e perspectivas, de modo a refletir as riquezas e especificidades da cultura brasileira. 